OPEN ACCESS

Edited by:

Richard Skarbez,

La Trobe University, Australia

Reviewed by:

Bas De Boer

University of Twente, Netherlands

Phil Lopes,

École Polytechnique Fédérale de Lausanne, Switzerland

${ }^{*}$ Correspondence:

Joakim Vindenes

joakim.vindenes@uib.no

Specialty section: This article was submitted to Virtual Reality and Human Behaviour.

a section of the journal

Frontiers in Virtual Reality

Received: 20 January 2021 Accepted: 24 March 2021

Published: 29 April 2021

Citation:

Vindenes $J$ and Wasson B (2021) A Postphenomenological Framework for

Studying User Experience of Immersive Virtual Reality.

Front. Virtual Real. 2:656423.

doi: 10.3389/frvir.2021.656423

\section{A Postphenomenological Framework for Studying User Experience of Immersive Virtual Reality}

\author{
Joakim Vindenes ${ }^{1,2 *}$ and Barbara Wasson ${ }^{1,2}$ \\ ${ }^{1}$ Centre for the Science of Learning \& Technology (SLATE), University of Bergen, Bergen, Norway, ${ }^{2}$ Department of \\ Information Science and Media Studies, University of Bergen, Bergen, Norway
}

Virtual Reality (VR) is a remarkably flexible technology for interventions as it allows the construction of virtual worlds with ontologies radically different from the real world. By embodying users in avatars situated in these virtual environments, researchers can effectively intervene and instill positive change in the form of therapy or education, as well as affect a variety of cognitive changes. Due to the capabilities of VR to mediate both the environments in which we are immersed, as well as our embodied, situated relation toward those environments, VR has become a powerful technology for "changing the self." As the virtually mediated experience is what renders these interventions effective, frameworks are needed for describing and analyzing the mediations brought by various virtual world designs. As a step toward a broader understanding of how VR mediates experience, we propose a post-phenomenological framework for describing VR mediation. Postphenomenology is a philosophy of technology concerned with empirical data that understand technologies as mediators of human-world relationships. By addressing how mediations occur within VR as a user-environment relation and outside VR as a human-world relation, the framework addresses the various constituents of the virtually mediated experience. We demonstrate the framework's capability for describing VR mediations by presenting the results of an analysis of a selected variety of studies that use various user-environment relations to mediate various human-world relations.

Keywords: user experience, virtual reality, postphenomenology, mediation theory, framework

\section{INTRODUCTION}

VR technologies are valuable and versatile tools because they allow for the instantiation of abstract ideas in encompassing virtual worlds. This capability of the medium enables us to simulate reality in a cost-effective manner, for instance by treating anxiety through exposure therapy in virtual environments (Lindner et al., 2019) or training surgery on virtual patients (Satava, 1993). Beyond mere simulation, however, VR also holds the power to realize goals in ways that would otherwise not be possible, such as reducing implicit racial bias by embodying white people in dark-skinned avatars (Banakou et al., 2016) or increasing self-compassion by changing perspectives through virtual embodiment (Osimo et al., 2015). This latter approach-realizing goals in ways that would otherwise not be possible-involves the design of virtual worlds with ontologies different than the 
real world, tailored to elicit a particular effect on the immersed user. The power of VR to change ourselves in this manner is usually attributed to the capability of the medium to induce a feeling of presence in the computer-synthesized worlds (Slater and Sanchez-Vives, 2016). Immersed in VR, the user is situated; she feels present in the virtual environment, experiences it from a particular point of view, embodies avatars and tools, and involves herself in the scenario or narrative of the application. From this mediated situatedness, where some possibilities for experience are left open while others are restricted, a particular subjectivity of the user is constituted in relation to the objectivity of the virtual environment. Consider, for instance, how the embodiment of a child-sized avatar constitutes the virtual environment as large and perhaps overwhelming, or how the embodiment as a victim in a scenario may constitute the world as an unjust world in need of change. The user experience of VR is in this way dependent on how the subjectivity and objectivity of experience are constituted in relation to each other. What makes VR practical for interventions, of course, is that although the user's subjectivity is constituted in relation to a virtual environment, the effects are not restricted to the bounds of the simulation. The experience also plays a role in effectuating an altered humanworld relation after exposure so that having experienced a virtual reality, reality itself is re-framed for the subject.

Because VR interventions owe their effectiveness to the experience of a virtually mediated subjectivity, we argue that insight into the phenomenology of these interventions can inform our understanding of them. In advocating for such a turn to experience, this paper presents a theoretical framework for understanding the user experience as mediated in relations constituted between user and environment. The mediation perspective that we advocate is distinguished from traditional approaches to understanding user experience in that it does not presuppose the human subject and the technology as poles between which interaction occurs (Verbeek, 2015a). Rather, it sees the human subject and the experienced technology as a result of this interaction as they "mutually shape each other in the relations that come about between them." (Verbeek, 2015a, p. 28). We purport this perspective is a more relevant way to understand the user experience underlying VR's capability to "change the self," as it specifically attends to how the human subject is mediated in the user-environment relation that is constituted.

\section{A Postphenomenology of Virtual Reality}

The framework we present for understanding and describing the virtually mediated experience is grounded in postphenomenology. Postphenomenology is a philosophy of technology that understands technologies in light of how they mediate human-world relations by co-constituting the subjectivity and objectivity of experience (Rosenberger and Verbeek, 2015). Postphenomenology is a highly relevant framework for understanding how VR technologies mediate experience, especially VR interventions, as these explicitly aim to change behavior, feelings, and attitudes, consequently, impacting the way that humans relate to their world. For instance, VR can be used to entice people to save for their retirement (Hershfield et al., 2011), enhance fear recognition in violent offenders (Seinfeld et al., 2018), or encourage prosocial behavior (Rosenberg et al., 2013). This is done by mediating a userenvironment relation in VR within which the experience that effectuates the intervention takes place. Usually, this experience is approached in research through measuring several aspects of it such as presence, confirming the virtual embodiment, measuring simulator sickness, and generally accounting for a select number of psychometric variables. In this paper, we argue that approaching experience qualitatively from a broader postphenomenological perspective can inform our understanding of the virtually mediated experience in a more holistic way than isolated constructs can offer. While a researcher studying user experience of VR from a post-phenomenological perspective would naturally also be concerned with whether a user feels present and embodied in the virtual environment, what she would have as her focus is how the embodiment and presence take part in constituting the user's subjectivity in relation to the objectivity of the environment. Approaching experience from a post-phenomenological perspective, therefore, does not involve replacing or rejecting established constructs used to measure experience; instead, it attends to this experience by describing it in terms of the subjectivity and objectivity arising from the mediation. For Immersive VR, this entails seeing the user experience as mediated in relations constituted between user and environment.

\section{Ethics}

Attending to the user experience of VR from a postphenomenological perspective can also be useful for ethical assessment. The post-phenomenological approach to ethics is one of ontological disclosure; it asks what kind of worlds we disclose through new technologies, and in the same manner, who we become in relation to these worlds (Introna, 2017). Therefore, it is by providing an increased understanding of the ways that VR technologies can mediate our experience that the post-phenomenological perspective can aid researchers in discovering potential ethical issues resulting from their designs. Ethical concerns are particularly relevant for VR interventions as they explicitly aim to affect human behavior. We know that VR owes the effectiveness of its interventions to its mimesis of reality; the benefits observed in studies "rely on the extent to which the experience is perceived as real" (Slater et al., 2020, p. 1). In addition to the shared phenomenology of presence (Loomis, 2016), reality and virtuality also share what Metzinger (2018) refers to as phenomenal transparency, where the medium takes a transparent role so that the content it presents is not subjectively experienced as a representation. Consequently, it is because VR experiences can be similar to real life experiences (Slater, 2009) that VR is a powerful technology that is capable of producing beneficent as well as non-beneficent results. How complex the ethics of VR may become upon mass adoption is not known. Madary and Metzinger (2016) argue that VR will change deeply established notions of who we are and how we identify and so "transform the structure of our life-world" (p. 2). What is clear, however, is that the powerful capabilities of VR to "change the self" require researchers to exercise ethical attentiveness to the various ways in which a participant's subjectivity can change as the result of experiencing a virtually 
constituted subjectivity. Although the content of the experience is virtual, the experience is "real as an experience" (Slater et al., 2020 , p. 5, emphasis in original), and the emotional and cognitive after-effects, although usually beneficial, can also be harmful (Slater et al., 2020). For instance, while VR interventions may reduce implicit racial bias (Banakou et al., 2016), they may also increase it in negative contexts (Groom et al., 2009; Banakou et al., 2020), suggesting potentially non-beneficent results when using VR as an "empathy machine." Similar warnings have been issued by Sri Kalyanaraman et al. (2010) who immersed participants in a simulation of the effects of schizophrenia. Although their simulation proved to be effective in increasing empathy and positive perceptions toward people who have schizophrenia in combination with non-VR perspective-taking exercises, they found that "mere exposure to a virtual simulation of schizophrenia by itself may not only be ineffective, but actually prove to be inimical..." (ibid, p. 441). Other non-beneficent results were also reported recently by Neyret et al. (2020) from a virtual recreation of a Milgram Obedience Scenario, who highlights it as "vitally important" to be aware of possible adverse outcomes resulting from virtual embodiment in scenarios-even if the chance of this occurring is deemed unlikely a priori.

Madary and Metzinger (2016) write how the embedding of VR in our world creates a "complex convolution, a nested form of information flow in which the biological mind and its technological niche influence each other in ways we are just beginning to understand" (p. 20). VR creates "not only novel psychological risks but also entirely new ethical and legal dimensions..." (ibid, p. 20). While no single approach or theoretical foundation can solve the ethical challenges of VR alone, we believe a qualitative turn to the user experience of VR-by inquiring into the experiential relationship established between user and environment-can be a complementary constructive angle from which researchers can uncover unintended effects resulting from their designs.

This paper is structured as follows. First, we provide a background to postphenomenology and account for its relevance as a framework for describing Immersive VR mediation. Having presented the paper's theoretical background, we detail our proposal of a post-phenomenological framework for understanding user experience in Immersive VR as mediated in user-environment relations. We demonstrate the applicability of the framework by analyzing a selected variety of studies on VR interventions that constitute particular user-environment relations in order to mediate particular human-world relations. After the analysis, we discuss the relationship between real and simulated subjectivity as well as the relationship between real and virtual worlds in more depth. Finally, we discuss the scope of the framework before outlining directions for future work to advance the applicability of the theoretical framework into the methodological.

\section{RELATED WORK}

\section{Postphenomenology}

The framework that we propose in this paper is informed by postphenomenology, a philosophy of technology that views technologies as mediators of human-world relations. With its phenomenological roots, postphenomenology understands humans and technologies as inseparable and views technologies as co-constituting human subjectivity and world objectivity (Rosenberger and Verbeek, 2015). Consider, for instance, how the embodiment of a car enhances the human being by constituting the subject as a driver and therefore also the world as more accessible or how, for a blind person, the white cane constitutes the world as such and extends the subject through the embodiment of the cane. Concerned with empirical data (Achterhuis, 2001), postphenomenology is pragmatic, and giving heed to its phenomenological origins, it draws its data from experience. Postphenomenology adopts from phenomenology the notion of intentionality as an invariant of experience: all consciousness is consciousness of something. Subjectivity and objectivity, experiencer and experienced-what Husserl referred to as the noesis and the noema-are two distinct ends of the polarity of experience. Postphenomenology stresses the role that technologies have in mediating this intentional relation by coconstituting both the human subject and their world. In doing a post-phenomenological investigation of a VR application, therefore, we would be interested in "who" the user becomes in relation to the virtual environment, and simultaneously, "what" the environment is for the user. In other words, we would be interested in what kind of user-environment relation is being mediated, but also beyond this, how the user-environment relation takes part in mediating the human-world relation outside of the virtually mediated experience.

Postphenomenology as a praxis-oriented phenomenology was established through the works of philosopher Don Ihde. An expanding group of scholars now contribute to the postphenomenological approach of studying the ever-expanding role of technologies in our lives, most notably Peter-Paul Verbeek, who extends Ihde's post-phenomenological thought in his theory of technological mediation (Verbeek, 2005a). In the sections below, we provide an account of Ihde's Human-Technology Relations before describing Verbeek's exposition of immersion as a human-technology relation.

\section{Human-Technology-World Relations}

Don Ihde identified four structures of human-technologyworld relationships (Ihde, 1990). The first of these he calls embodiment relations, where the combination of human and technology together relate to the world. In embodiment relations, there is transparency, as when we look through our eyeglasses or talk through the phone. Second, he discusses hermeneutic relations, where humans "read off" an abstract representation by a computer, such as a weather forecast or an MRI scan. Third, in alterity relations, humans interact with technology directly within its own system, a common example being interaction with an ATM or a calculator, where the world withdraws into the background. Lastly, Ihde (1990) discusses what he calls background relations, where the technology is an implicit condition affecting the environment, partly serving as the context in which we find ourselves (e.g., an air conditioner). Ihde (1990) illustrates his embodiment, hermeneutic, alterity, and background relations through diagrams indicating on 
TABLE 1 | Human-Technology Relations Diagram (Ihde, 1990).

\begin{tabular}{ll}
\hline Embodiment relation & (human - technology) $\rightarrow$ world \\
Hermeneutic relation & human $\rightarrow$ (technology - world $)$ \\
Alterity relation & human $\rightarrow$ technology (world $)$ \\
Background relation & human $\rightarrow$ (technology / world $)$
\end{tabular}

Arrows indicate intentionality.

which poles, subjective or objective, the technology primarily is "situated" with arrows indicating intentionality, as seen in Table 1.

\section{Immersion as Human-Technology Relation}

The human-technology-world relations identified by Ihde are not so exhaustive as to include all possible relations. Verbeek has further identified several human-technology relations enabled by newer technology developments, where the immersion relation is the most relevant for the user experience of VR. The immersion relation can be understood as a more active version of Ihde's background relation, where the environment and the technology become merged (Verbeek, 2015b; Aydin et al., 2019). It is more active in the sense that the environment is aware of human beings and actively interacts with them. The result is that human beings are directed toward technologies, and the technologies are in turn directed toward them, resulting in a "reflexive intentionality" (Verbeek, 2005b) where humans can have new relations toward themselves through the technology. Although this relation is referred to as an "immersion" relation, we should note that Verbeek does not use the word "immersion" in order to relate it to VR technologies in particular. As examples of immersion relations, Verbeek (2011) describes smart toilets that analyze excrement and provide health reports, or beds that can detect whether somebody falls out. The immersion relation is nevertheless relevant for understanding VR because VR technologies open entirely new possibilities for reflexive intentionalities, which we return to in our analysis.

\section{User-Environment Relations}

Having described Ihde's and Verbeek's human-technology relations, we might ask what kind of relation VR constitutes. As we have discussed, the benefit of VR is its flexibility; it can be adapted to unique situations and be designed to elicit vastly different effects. In this regard, VR can be said to be an extreme meta-medium (Kay and Goldberg, 1977), as virtually all other media can be reproduced within it, including future, non-existing media. The result is that " $\ldots$ each form of VR is a medium unto itself.” (Lanier, 2017, p. 204). For this very reason, any attempt to give a total account of the various possibilities of VR mediation is impossible; all the various human-technology relations introduced above could conceivably be had within various VR applications. There is an invariant human-technology relation that lays the ground for other relations within the virtual, however, it takes a special form in VR. Comparing immersive VR to non-immersive simulators, Voordijk and Vahdatikhaki (2020) write that "when the technology 'disappears' in embodiment, the role of the VR simulator changes, in terms of Ihde, from an alterity relationship to an embodiment relationship.” (p. 10). While the VR HMD becomes transparent in use and we act through it, the intentional relation is not mediated toward the world, rather, it is mediated toward the virtual environment. Consequently, when embodied, the user is in an alterity relation toward the virtual environment, interacting directly with the technology within its own system. Thus, in the embodiment of a VR HMD, we act both through it and upon it, which is why VR can simultaneously mediate both (i) the objectivity of the environment in which users are situated (alterity) as well as (ii) the users' subjective position and relation toward that virtual environment (embodiment). So, while we embody parts of the VR technology (hardware, avatars, tools) as part of our subjectivity in a transparent embodiment relation, the objectivity of our experience (environment, actors, social scenarios) is also mediated by the same VR technology, constituting an opaque alterity relation in which the world is in the background. This human-technology relation that VR constitutes, we describe in our framework as user-environment relations. This embodimentalterity relation can be schematized in the manner of Ihde (see Table 1) as follows:

$$
(\text { Human - Technology }) \rightarrow \text { Technology }(- \text { World })
$$

This schema denotes a user-environment relation: a human in an embodiment relation with the technology (i.e., the user) in an alterity relation to the technology (i.e., the environment), while the world is in the background.

\section{A POST-PHENOMENOLOGICAL FRAMEWORK FOR IMMERSIVE VIRTUAL REALITY}

Immersive VR mediates user-environment relations in which the embodied user stands in an intentional relation to the environment while the world is in the background. This humantechnology relation that VR constitutes lays the ground for our framework of VR mediation. In substantiating our framework, this section will present and discuss the constitutive elements of this mediation process in more depth. As illustrated in Figure 1 [which is an altered version of Figure 3 by Hauser et al. (2018) depicting the roles of design researchers in RtD inquiries] this means recognizing the subjectivity-objectivity structure as constituted within VR (the user-environment relation), as well as the subjectivity-objectivity structure as constituted outside of VR (the human-world relation).

Our framework mirrors the overview of technological mediation provided by Hauser et al. (2018); the humans of the study, the mediator, and their world are the basic constituents of any technological mediation process (see Figure 2). As the technology mediates the humans' subjective relation to their worlds, who these people are, where they are situated, and what the technology/mediator is are essential overarching variables in understanding technological mediation post-phenomenologically (Hauser et al., 2018). In postphenomenological inquiries in Human-Computer Interaction, the researchers stand in constructive roles regarding the studying, 


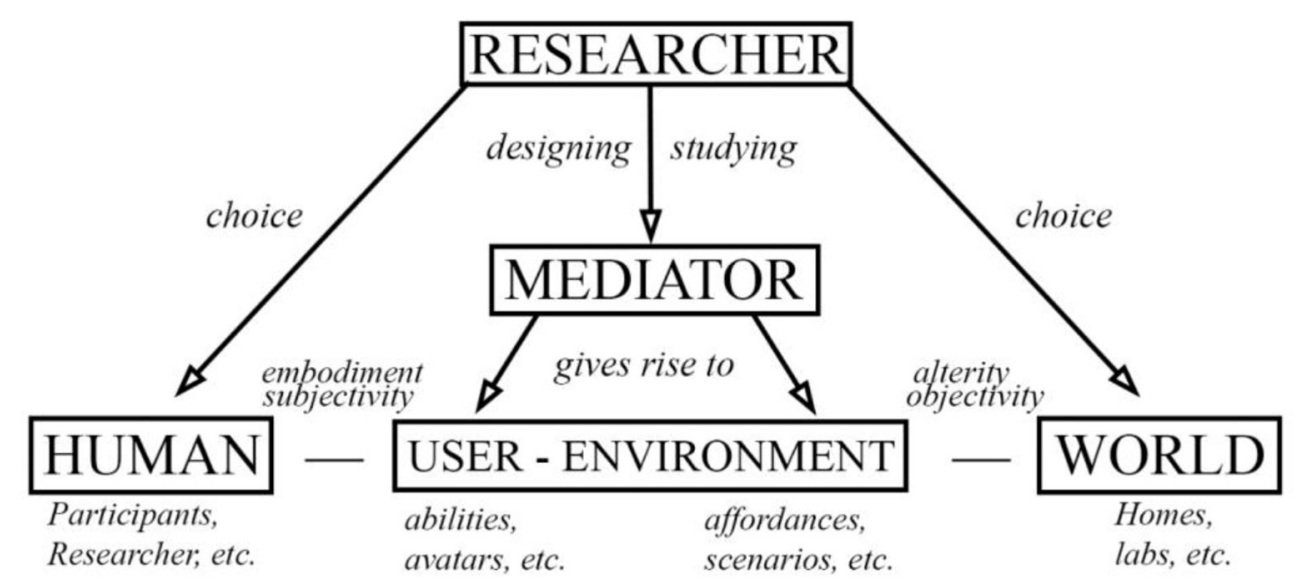

FIGURE 1 | Illustration of our Post-phenomenological Framework for Studying User Experience of Immersive VR as Mediations.

\section{How the world is there or becomes meaningful for humans - hermeneutic}

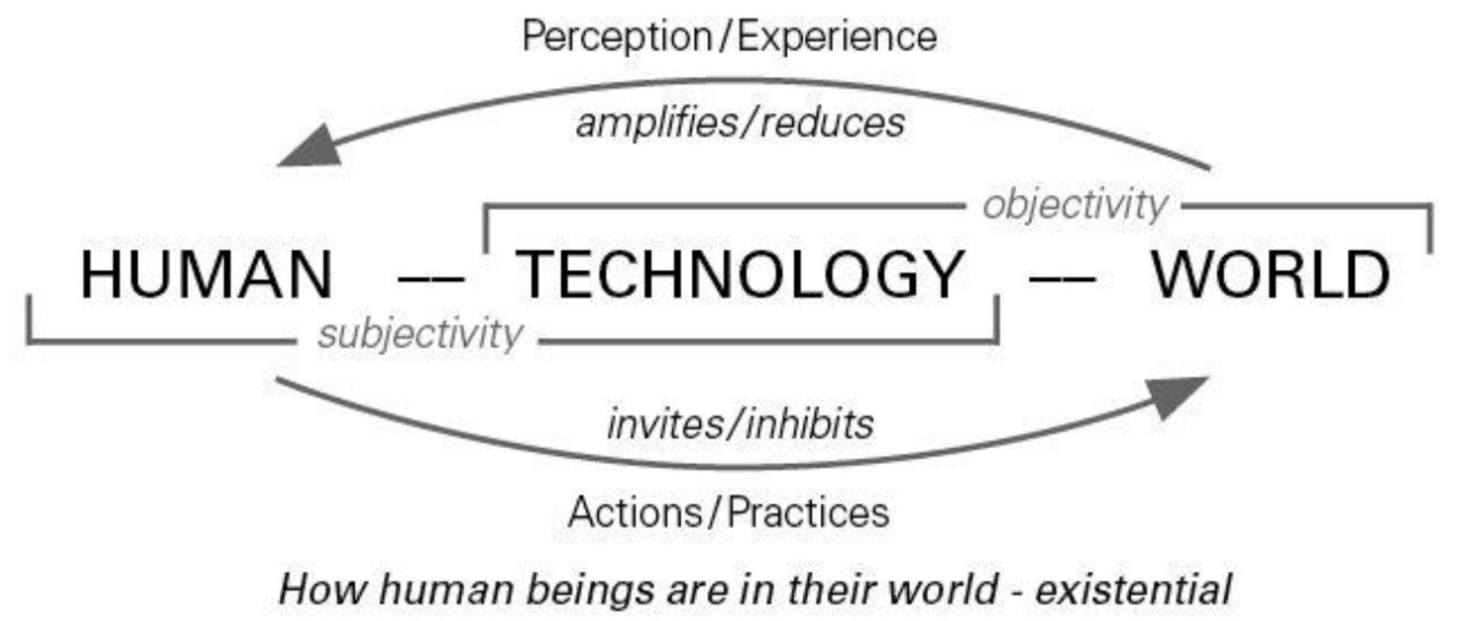

FIGURE 2 | Technological mediation (Hauser et al., 2018).

choosing, and designing of these constituents (see Figure 3). The next two elements in our framework more concretely address the user-environment relation: what occurs when a human participant engages with the VR application. The VR is here a mediator that gives rise to (4) a User, and (5) an Environment; the human as user has an altered subjectivity constituted in relation to the virtual environment.

In the next sections, we detail the various elements of our framework. An overview of the framework components is provided in Table 2.

\section{Human}

The first element of the framework is concerned with the human who engages with the VR mediator. Postphenomenology sees technologies as multi-stable, the same technology can have several different stabilities in terms of how it is used and experienced. While multistability of technology can be actively designed for-our best example being the smartphone, the modern swiss army knife-multistability is also present in cases where the intention is for the artifact to embody a concrete function, such as a VR application intended to deliver a particular intervention effect. In short, technologies "simply can't be reduced to designed functions" (Ihde, 2002, p. 106). As a classical example, hammers are made for hammering nails, but can find other stabilities, such as being a paperweight or a weapon (Ihde, 2002). In the same way, an interactive VR application is not fixed in how it can be "used" or experienced, the user-environment relation that is mediated depend not only on the VR application, but on the individual human who engages with it in their context of use. The particularities of this group, such as their sedimented or unestablished relationship with VR technology, or their attitudes toward technology in general, will impact their virtually 


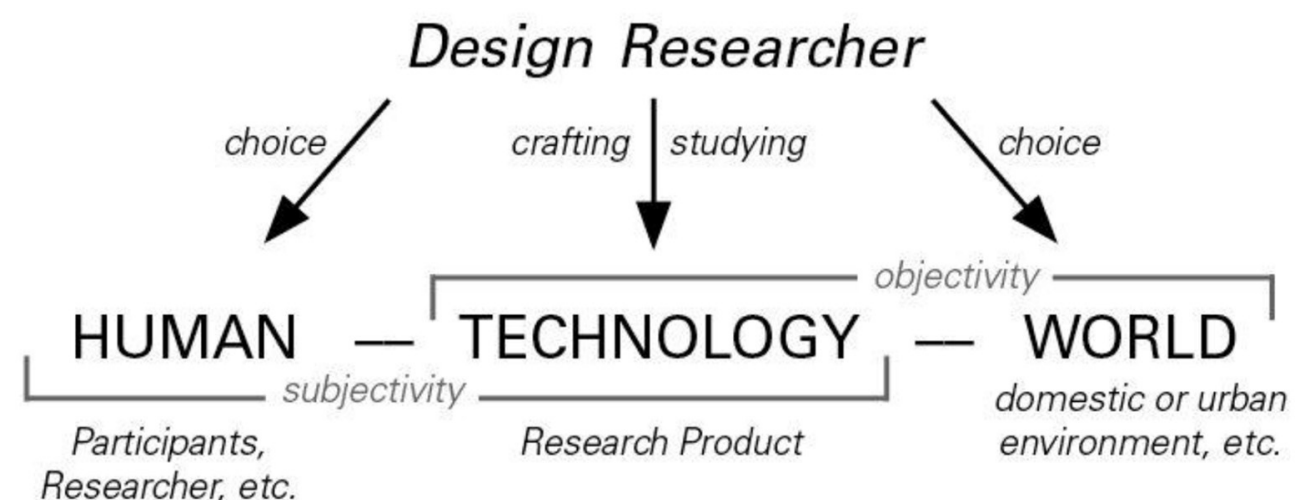

FIGURE 3 | Roles of design researchers in their RtD inquiries (Hauser et al., 2018).

TABLE 2 | Overview of Framework Constituents.

1. Human. The human being partaking in the study. Here, the particularities of the person might be mediated, as well as impact the mediation. Examples of human factors: personality, gender, socio-economic status, interests and motivations, involvements, and previous technology experience. Human factors vary and impact relational and hermeneutic strategies toward the technologies

2. World. The use context of the application where the VR application is being used. This constitutes the background of the VR experience. Here, also, the particularities of the context might be mediated, or take part in mediating the VR experience. Examples: hospital, lab, work, or domestic settings

3. VR Mediator. The VR application that is being designed or evaluated for intervention purposes. Designed or studied for its ability to provide an experience or user-environment relation that can be a catalyst for change

4. User. The human as user in an embodiment relation to the alterity of the virtual environment. The user subjectivity is in a nested relation to the subjectivity the human individual has in relation to her actual world (Gualeni and Vella, 2020) but is further affected by avatars, tools, interaction possibilities, position, involvements, and social scenarios

5. Environment: The virtual environment as experienced by the user during the VR embodiment. The part of the VR application that is not embodied by the user, but is rather acted upon, or that which acts upon the user, including social actors, 3D objects, events, etc.

mediated experience. These are the humans who will experience the mediating effects of the technology on their self as well as on their world. Professional skills or diagnostic criteria fall under this point, but also differences in experience, culture, gender, etc., as this may be indicative of different relational strategies in approaching the technologies. Relational strategies can be defined as particular understandings and bodily approaches that "enables a user to relate to a technology in terms of a particular stability" (Rosenberger and Verbeek, 2015, p. 29). Similarly, different people may have different hermeneutic strategies, strategies which "enable a user to apprehend the meaning of a technology's readout in terms of a particular stability..." (Rosenberger and Verbeek, 2015, p. 29). This does not mean that VR applications can be so multi-stable that any user-environment relation can be experienced; as Ihde (2002) notes, "Multistability is not the same as neutrality." (p. 106). All technologies, however open they may be, have a certain directedness (Verbeek, 2008). Despite there being various trajectories for use, these are not unlimited, and some of these will prove more dominant and stable than others.

\section{World}

The second element of the framework is concerned with where in the human sphere the mediating technology is used. Phenomenological accounts of places and situational contexts highlight the inseparability of humans from their environments (Donohoe, 2017). Places-understood geographically, architecturally, or socio-culturally-take part in shaping behavior, identity, and moods; places, then, can also be regarded as mediators of our selves. The humans in the study, therefore, are only one aspect; equally relevant is the "world" in which these humans will use the technology. The world is the "use-context" of the application and will be present for the user as the background of experience, although she is immersed in a virtual world. Again, we return to the postphenomenological concept of multi-stability; technologies will have different meanings for different people in different contexts. While it is possible to evaluate an application experimentally in-lab that is originally intended to be used elsewhere, this is not as likely to give an account of what the mediation effects of the technology will in fact be, simply because one of the constituents of the virtually mediated experience (the world) will be different than what is being evaluated. This is equally due to the situational context (being observed by researchers in-lab) as the geographical context of being in the lab. The use-context affects the experience of the virtual environment and the virtual environment may also further mediate how the participant sees the use-context.

\section{Mediator}

The third element in the framework is the technology, or mediator, which within VR interventions usually comprises a virtual environment that is experienced from the first-person point of view. The design of this technology can have various goals-such as therapy or training - which is meant to be attained by immersing the human into the virtual environment. This mediator gives rise to a user-environment relation: the human 
becomes an embodied user, immersed in, and standing in an intentional relation to, the virtual environment, while the world is in the background. In order to describe the mediator element more thoroughly, we detail the mediations that it gives rise to in the next sections: (4) User and (5) Environment.

\section{User}

The first sub-element of the mediator is concerned with the embodied user, situated and operating from a particular subjective position within the simulation. The "user" entity is not the same as the human participant, nor is it merely the subjective position into which the participant is immersed. Rather, it is the human participant as user, i.e., the human participant under active mediation of the VR technology, virtually embodied and in an intentional relation to the virtual environment. The subjectivity of the user, therefore, can be said to be "in a nested relation to the individual's subjectivity in the actual world" (Gualeni and Vella, 2020, p. xxi). Human subjectivity is being mediated by the VR application, within the simulation in relation to the virtual world (User) as well as outside the simulation in relation to the real world (Human). This is an example of what De Mul (2010) refers to as poly(ec)centric positionality, denoting a mediated multiplication of one's center of experience. Phenomenologists such as Merleau-Ponty (2002) have famously distinguished between the body as lived and the body as object. This holds for VR also; while our avatar can be seen as one $3 \mathrm{D}$ object situated amongst others, it is also, to the degree that it is embodied, that through which we experience. Ihde (2002) refers to Merleau-Ponty's lived body as body one and understands the objectified body two as the acted-upon body of Foucalt; "... body one permeated with the cultural significances that are also experienced." (p. xviii). According to De Mul (2010), it is this eccentricity-our being simultaneously inside (subjectivity) and outside (objectivity) of ourselves - which is the condition for telepresence and VR. With these technologies, we can objectify our thoughts of who we want to be, and, through embodiment, we can experience reality from the perspective of these bodies. In poly $(\mathrm{ec})$ centric positionality, the virtual constitutes "a complete and additional, artificial experiential center" (Gualeni, 2015, p. 115 ) which lays the foundation for the simulation's capability to "elicit ontological effects" (ibid, p. 118).

The question of the user element in the framework is how this new, artificial experiential center is experienced during embodiment. It is concerned with what kind of subjectivity is mediated within the user-environment relation. For instance, what avatars and tools does the user embody? How is she positioned in relation to the virtual environment, and what are the possible points of action from this situatedness? Is she involved in a certain story, scenario, or task? Here, examples may range from leading a team of surgeons, to being positioned as a victim of physical abuse. What the user can do, and who she experiences herself to be, is defined in relation to the virtual environment and the affordances it presents.

\section{Environment}

In strong relation to the user, therefore, is the environment, the second sub-element of the mediator. The environment represents the part of the VR application that is not embodied, and therefore, that to which the embodied user relates to as alterity. In focus is the question: in what kind of environment or world is the user situated? What are the basic parameters for how this environment works and what it represents? For instance, the system may display some objects as interactable and some merely acting as decorative or situational elements, some in the proximity of the user and some at a distance. Such choices are a part of the intended mediation of the researcher, impacts the subjectivity of the user, and provides the technology with a certain directedness (Verbeek, 2008). It is here important to note that we understand the environment (post)phenomenologically. We are interested in how the environment is understood from the situated standpoint of the user, not from a detached God's eye view. Similar questions exist here as for the world constituent, but in relation to the environment. For instance, as what is this virtual environment disclosed for the subject? What are the most apparent features or affordances of this environment, and what does this communicate to the user? Does the environment invite certain trajectories of action, while inhibiting others? In other words, we are interested in how the virtual environment is experienced in its relation to the user, that is, how the human perceives the environment when immersed and embodied. How the environment is experienced is not just dependent on the objective features of the environment. An illustrative example of such numerous convoluting, mediating factors is the various virtually reconstructed Milgram Obedience Scenarios (Slater et al., 2006; Gonzalez-Franco et al., 2018; Neyret et al., 2020). In these virtual recreations of the famously controversial research described by Milgram (1964), participants meet with real researchers in a lab who do an experiment, but the experiment is to meet virtual researchers and partake in their experiment in a virtual lab. In the event of partaking in such a study, participants are in a very real sense both real and virtual participants, and likewise, stand in relation to both the real researchers and the virtual researchers. VR technologies are not immersive to the extent of the participants forgetting their normal feeling of self or their worlds. The real world is still present as a background relation, and the user subjectivity is in a nested relation to the subjectivity of the human individual in the real experiment.

\section{ANALYSIS OF USER-ENVIRONMENT RELATIONS}

VR constitutes an embodiment-alterity relation that we describe as user-environment relations, where the embodied participant is in an intentional relation toward the alterity of the VR application. This describes VR mediation generally; how a particular user-environment relation is mediated depends on what is embodied (subjectivity), and what is related to as alterity (objectivity). In order to concretize our framework, this section presents an analysis of various user-environment relations constituted in VR interventions. What we intend with our analysis of user-environment relations in VR is to account for some observed variance of how user-environment relations can 
TABLE 3 | Overview of User-Environment Relations from Analysis.

Simulated Subjectivity: Simulated Subjectivity refers to mediations where an altered subjectivity is experientially pronounced; a simulation of "what it is like." This may be done with the intent of having the application act as an empathy machine or for the application to facilitate for the experiencing of new first-hand perspectives on known information. Examples include what it is like to suffer from strokes, schizophrenia, blindness, as well as the effects of psychedelic drugs

Simulated Objectivity: Simulated Subjectivity refers to mediations where the user is "transported" to a new place, where there is little to no explicit attempt to alter user subjectivity apart from being immersed in the environment. Examples include medical simulations, cultural heritage, VR exposure therapy, etc.

Subjectivity-Objectivity Inversion: Subjectivity-Objectivity inversion refers to a mediation process in which the subjectivity-objectivity poles are inversed, for instance framing the Self as Other, or the Other as Self to change either one's perspective on one's self or one's perspective on others. What "other" is being re-framed (opposite gender or different age/race/socio-economic status) varies depending on the Human-World Relation the researchers intend to achieve (for instance increased empathy or less racial bias)

Subjectivity-Objectivity Synchronization: Subjectivity-Objectivity Synchronization refers to a mediation process in which the subjectivity and objectivity of experience approximate each other toward a state of equilibrium. This can be initiated by mediating properties of the subjectivity to affect the objectivity or the other way around. Which mirrors which can depend on what Human-World Relation the researchers intend to achieve or measure

be structured. The research papers in the analysis were selected in order to display the breadth of ontological structuring that is possible within the overarching embodiment-alterity relation. The analysis highlights in post-phenomenological terms how the interventions constitute various user-environment relations in order to mediate various human-world relations.

We categorize the identified user-environment relations as follows: (1) Simulated Subjectivity, (2) Simulated Objectivity, (3) Subjectivity-Objectivity Inversion, and (4) SubjectivityObjectivity Synchronization. The first two categories focus on the two distinct poles of experience in VR: subjectivity (embodiment) and objectivity (alterity). These are discussed rather briefly, and by dealing with subjectivity and objectivity in isolation, these categories also act as an introduction for the two latter categories where subjectivity and objectivity are more entwined. Consequently, the analysis is mainly concerned with the two latter categories, "Subjectivity-Objectivity Inversion" and "Subjectivity-Objectivity Synchronization", as these describe the novel relations that can be constituted between user and environment in VR. A summary of the identified userenvironment relations is provided in Table 3.

\section{Simulated Subjectivity}

In providing an experience, VR mediates sensory stimuli, some of which is embodied and becomes "part of" the user, and some of which is not embodied, and as such stands in an alterity relation toward the user as an environment. While this means that all VR applications will necessarily simulate both subjectivity and objectivity, what is novel or unique in the VR experience may be more pronounced experientially for the user. Simulated subjectivity, therefore, refers to cases where the intended mediation is to convey what it is like to be another (subjectivity), with less focus on mediating a particular virtual environment (objectivity). It refers to cases where it is intended for the mediation of subjectivity to be more pronounced experientially than the objectivity. As an example, Suzuki et al. (2017) developed the Hallucination Machine by processing panoramic videos using Google's Deep Dream AI, in order to "[induce] visual phenomenology qualitatively similar to classical psychedelics." (p. 1). Other examples of simulated subjectivity include simulations of various visual impairments in VR (Ahn et al., 2013; Ates et al., 2015; Jones et al., 2020) as well as strokes (Maxhall et al., 2004) and schizophrenic episodes (Nyre and Vindenes, 2020). Simulating subjectivity is naturally linked to empathy as it could be said to be a virtual representation of "what it's like to walk a mile in someone else's shoes." However, most of the interventions promoting empathy in our analysis are discussed under section Subjectivity-Objectivity Inversion, as their strategy toward generating empathy is by mediating a more reflexive user-environment relation in which the alterity/objectivity is also of importance.

\section{Simulated Objectivity}

As the inverse of Simulated Subjectivity, Simulated Objectivity refers to mediations when the participant is immersed in an environment or scenario (objectivity) where there is no explicit intention of altering user subjectivity. Typical examples here include simulator training for various purposes such as surgery (Alaraj et al., 2011), but can also be exemplified through virtual field trips (Çaliskan, 2011), cultural heritage (Rua and Alvito, 2011), or VR exposure therapy (Flobak et al., 2019). In these cases, the success of the simulation is dependent on the degree to which the simulation represents reality. This is VR as it perhaps is traditionally understood, where the participant is "transported" to an environment but remains "herself." Thus, there is the intention of keeping the participant's subjectivity more or less non-mediated, apart, of course, from the mediating effects of the environment/situation itself.

Having briefly described Simulated Subjectivity and Simulated Objectivity as the two distinct poles that can be targeted in mediation, we move on to the reflexive user-environment relations, where the structured relationship between subjectivity and objectivity is of importance. Naturally, the two next userenvironment relations also include the simulation of subjectivity and objectivity, but here it is the user-environment relations that are highlighted.

\section{Subjectivity-Objectivity Inversion}

In this section, we present a user-environment relation that we refer to as a subjectivity-objectivity inversion. We discuss this from two angles: mediating the Other as Self and mediating the Self as Other.

\section{Other as Self}

As humans, we identify in particular ways. We identify as individuals, but also with particular groups, such as sociocultural, racial, and ethnic groups, as well as gender and age. To various extents, other groups are experienced as such, other, and so we experience ourselves and our own situation in a different perspectival manner than we do others and their situations. 
While this is a natural limitation of being a particular human being, VR can allow a user-environment relation that constitutes what has traditionally been related to as Other (objectivity) as Self (subjectivity). The studies which we cite below as examples of this usually comprise an active instantiation of perspective-taking (van Loon et al., 2018) where VR allows the point of perspective to be an actual experiential center as opposed to one imagined through cognitive activity.

An example of such a subjectivity-objectivity inversion, Other as Self, is present in the study by Banakou et al. (2016), who embodied 90 white females in black virtual bodies. They found an immediate decrease in implicit racial bias against black people. A similar experiment was performed by Hasler et al. (2017) who embodied 32 white females and 32 black females in avatars of various color so that, over two sessions, all participants had been embodied in both black and white avatars. They found that the embodiment enhanced mimicry of behavior between those of the same embodied racial group-independently of the actual race. Similar role changing by means of virtual embodiment has been conducted by Seinfeld et al. (2018) who embodied male domestic violence offenders in virtual female bodies where they experienced a virtual scene of abuse from a first-person victim perspective. After exposure, the male offenders had an improved ability to recognize fear in female faces, a trait which offenders as a group score significantly lower on compared to controls (Seinfeld et al., 2018). Other examples include embodying adults as children (Tajadura-Jiménez et al., 2017; Hamilton-Giachritsis et al., 2018), embodying younger people as elderly (Hershfield et al., 2011; Banakou et al., 2018), or even embodying animals (Ahn et al., 2016). In all these cases there is a perspectivetaking where what was traditionally conceived of as outside one's subjectivity enters within it. What this "other" should be depends on the kind of intervention that is intended. Mothers may get an increased understanding of what it means to be a child, which in turn may alter how they view their role as mothers. Younger people may experience what it is like to inhabit an aged body, perhaps altering how they view the impermanence of their youth and the role of their elders, and people embodied as animals may feel more connected to nature by being directed to reflect on the fact that animals are sentient too. By reframing what is mediated as the subjectivity and objectivity of experience, VR can through subjectivity-objectivity inversion help humans bypass sedimented relations and facilitate a perspective-taking that is more directly experienced.

\section{Self as Other}

Another example of subjectivity-objectivity inversion is the reframing of the Self as Other. Just as being a particular human being comes with a limited perspective of others, seeing ourselves from our own point of view can have its limitations as well. "From the perspective of the self, the other is so rounded out that it is a consummated, self-sufficient whole. In contrast, the self cannot see itself in that way. It is tied up in the incompleteness of its own story..." (McCarthy and Wright, 2004, p. 75). While we may be able to see others for who they are now, we see ourselves in terms of both our future and our past. Being caught up in worries for the future and regrets from the past may cloud our access to the present reality. Objectifying the self, therefore, may come with its own benefits of altered perspectives. The studies cited below usually comprise a more active instantiation of selfdistancing theory (Leitner et al., 2017) of which methods are traditionally performed through the imagination. An example here is the study presented by Osimo et al. (2015), who had male participants embodied in avatars closely resembling themselves describe a personal problem to a virtual person in the likeness of Dr. Sigmund Freud. When the participant has described his problem, his body is swapped to that of Freud's, now seeing the avatar created in his likeness, which he previously identified with, sitting opposite him. Then his avatar begins to tell the story he had just told back again to the user embodied as Freud. Here, the participant as Freud again answers in terms of advice, before swapping back to the avatar again, and so on. In this way, the application reframes the self as other, as well as the other as self, and ideally allows the user to address his own problems as he addresses others' problems. Osimo et al. (2015) write how “...this form of embodied perspective-taking can lead to sufficient detachment from habitual ways of thinking about personal problems, so as to improve the outcome, and demonstrates the power of virtual body ownership to affect cognitive changes" (p. 1). A study similar in mechanism was conducted by Falconer et al. (2014) where female participants were trained in providing a compassionate response, which they delivered to a child in VR while embodied in a (non-lookalike) adult body. Later, the participants experienced their own compassionate statements in the embodiment of a child, which the researchers found increased self-compassion and feelings of being safe. Here, the perspectivetaking which the body-swapping facilitated (i.e., the alteration of subjective roles) allowed the participants to be both on the giving and receiving end of compassion. Another example is brought forward by Bourdin et al. (2017) who created out-ofbody experiences in VR by embodying participants in avatars, and changing the viewpoint so that they could view their virtual bodies from outside, reducing fear of death in the participants. Our final example of a subjectivity-objectivity inversion is the embodiment of participants as older versions of themselves in order to promote saving for their retirement (Hershfield et al., 2011). Here, the participants embody their future selves as part of their subjectivity and look in a virtual mirror. What is "other" in this intervention, however, and which the researchers intended the participants to identify more strongly with, is the aging of this future self. This can also be done where the "other" is not age deterioration, but increased/decreased physical fitness in order to increase motivation (Fox and Bailenson, 2009).

In the user-environment relation we call subjectivityobjectivity inversion-self as other and other as self-what the human participant embodied as user relates to as themselves is inversed. The result is that what was previously embodied (subjectivity) is now the alterity (objectivity), or that what was previously alterity is now embodied. This makes for an immersion relation between the user and the environment which constitutes a reflexive intentionality where the user can experience standing in new relations to themselves and others. We reiterate that reflexive intentionalities occur when the human is in an intentional relation to the technology-infused 
environment, where the technology-infused environment is also directed in intentionality toward the human. The human can experience how the environment perceives or interprets her from its perspective. In VR, however, the reflexive intentionality is realized somewhat differently. Firstly, the technology is fused with the environment in the sense that the technology is what instantiates the environment as such. Further, the environment does not abstract or convey a "representation" to the user of how it perceives her, which the user is meant to see from her situated perspective. Instead, aspects of the virtual environment that the user stands in an intentional relation to, such as a social actor, can itself be embodied so that the new relation that is opened toward one's self can be experienced more directly.

\section{Subjectivity-Objectivity Synchronization}

Having described Subjectivity-Objectivity Inversion, we turn to the case of Subjectivity-Objectivity Synchronization. A subjectivity-objectivity synchronization is an attempt at producing harmony between the inner life of the user and the external world that is experienced. The attempt can either be to make the inner life of the user be represented through the external world, or to make the external world affect the inner life of the user, or both. In the way that subjectivity-objectivity inversion utilizes an active instantiation of perspective-taking and/or self-distancing techniques, applications facilitating subjectivity-objectivity synchronization actively instantiate meditative techniques such as Mindfulness. Many meditation or relaxation techniques have as their aim to redirect focus and attention on the breath or the body in order to promote a feeling of union both with oneself and the world. In VR, the attempt to promote unity between subjectivity and objectivity-self and other-is approached explicitly by blurring distinctions or creating new relationships between the two. For instance, Roo et al. (2017) created a mixed reality sandbox where the user can create a virtual environment by restructuring sand in a physical sandbox. The sandbox has an overhanging depth sensor measuring the peaks and valleys of the sandbox, and a projector that projects visual terrain upon it. Having created the environment, the user can immerse herself in a $3 \mathrm{D}$ render of this world through an HMD where the environment responds to physiological data of the user, such as breath and heart rate. Here, the aim is to facilitate mindfulness meditation through a focus on the body as it is mediated through the environment. The mediation amplifies the focus on bodily sensations such as breath and heart rate, and by having this represented in the external environment, the otherwise clear-cut boundary between self and other is diminished so that there is subjectivity in objectivity and vice versa. A similar example is brought by Amores et al. (2019) who designed "Deep Reality, a VR experience of underwater fluorescent beings that move based on biometric information such as electroencephalogram (EEG), heart rate (HR), and electrodermal activity (EDA). The aim was reflection and relaxation. Here, again, the recurring pattern is that of changing the external environment to affect inner states, and as with Roo et al. (2017), the external environment is in turn based on inner states or approximations of these, constituting a neurofeedback loop in which it is intended that the subjectivity and objectivity of experience should approximate each other toward a state of equilibrium. Another example is brought forth by Stepanova et al. (2020) who designed JeL, an immersive VR system designed "to bring awareness to our physiological rhythm, fostering a connection with our bodies, each other, and nature (p. 641). Here, two users aim to synchronize their breath in order to grow corals in a coral reef. Other examples include the projection of artistic visualizations in VR based on EEG in order to induce positive pre-sleep (Semertzidis et al., 2019), biofeedback through projection to support yoga-breathing practices (Moran et al., 2016), and virtual environments generated by users' brain activities and respiratory rates in order to assist novice users in learning to reduce stress through mindfulness mediation (Prpa et al., 2016).

In these user-environment relations, the users also stand in an intentional relation toward the environment and so experience the environment, and likewise, the environment is in an intentional relation toward the user and "experiences" the user. In the study by Semertzidis et al. (2019), for instance, where the EEG is artistically visualized, the user perceives how the mediator interprets her state. This makes for an immersion relation between the user and the environment and opens up for a reflexive intentionality where the user not only experiences the environment, but a new perspective is opened toward one's self. Depending on the extent to which the user attempts to read or interpret the "message" of the application, these relations may lean toward hermeneutic as opposed to alterity.

This concludes our analysis of user-environment relations in VR interventions. We wish to stress that this list is far from exhaustive, and that the user-environment relations do not necessarily exclude each other. It is perfectly possible to imagine combinations of these as well as other possible subjectivityobjectivity configurations. We return to the idea of VR as an extreme meta-medium: each VR application constitutes its own form of medium. Beyond what we have described above, every user-environment relation will have its own subtly differently constituted subjectivity-objectivity structure, and we expect more nuances and complexity as researchers relate to actual phenomenological accounts. As Ihde (2012) writes regarding the methodology of phenomenological investigations, "[t]he analysis begins with what appears (noema) and then moves reflexively toward its how of appearing [noesis]" (p. 31). What kind of subjectivities will be revealed in virtual worlds cannot be grasped beforehand; this is rather discovered reflexively based on the mediated experience.

\section{DISCUSSION}

Interaction with technology is traditionally understood as something that happens between the human being and the technological artifact (Verbeek, 2015a). In contrast, postphenomenology takes the perspective of understanding the human subject and the technological artifact phenomenologically as they arise from the interaction; it pays attention to how the human subject and technological artifact mutually shape each other in the relation that comes between them. The perspective 
sees the design of technological objects as also involving "the design of human subjects who interact with these objects." (Verbeek, 2015a, p. 28), making it particularly relevant for understanding the user experience of VR interventions whose aim it is to "change the self." The theoretical framework in this paper is proposed as relevant for describing both intended and actual VR mediations. In order to clarify the contribution of the framework, we discuss more in depth the relationship between real and "virtual" subjectivity, as well as real and virtual worlds, before discussing the scope of the framework. We end the discussion by outlining directions for future work of advancing the applicability of our framework into the methodological.

\section{The Relationship Between Human and User}

Attempting to understand the nuances of the fleeting and mediated experience of VR can be complex. VR is a personal experience and will alter (and depend) on who the participant is, and in which world of meaning that they live. While the VR application is constant, the lived VR experience is a transaction between the technology and the human. So how exactly is this relationship constituted? To draw an example from post-phenomenological literature, Kaposy (2017) looked at how simulating ethical scenarios in medical education purports a view of the medical student more as an object than a subject. Utilizing Ihde's distinction of body one and body two-body one being the subjective, lived body, and body two, the objectified social and cultural body - the insight by Kaposy (2017) is that the students within the scenario are being evaluated after certain objective criteria, constituting an expected way of being that is abstracted as an object body. This is also the nature of interventions in Immersive VR. Within the design, the role that is more or less adopted upon embodiment and defined in relationship to social actors and the virtual environment is an abstract object body, a "body two." We draw on information from our environment and our bodies' appearance in determining who we are, and this impacts our behavior. This is, of course, not just a phenomenological discovery. This nested subjectivity is also described within other disciplines. For instance, both The Proteus Effect (Fox et al., 2013) and the idea of Body Semantics (Slater and Sanchez-Vives, 2014) claim, and demonstrate, that body type can influence attitudes and behaviors. The Proteus Effect describes the mechanism utilized in many VR interventions from a social psychological perspective based on self-perception theory, where participants conform to the behavior they imagine that a third party would expect (Slater and Sanchez-Vives, 2014). Body semantics approaches this from a neuroscientific perspective and sees this as an intrinsic property of brain functioning, where the brain generates attitudes and behaviors "concomitant with that type of body, independently of any other factors such as social expectation." (Slater and Sanchez-Vives, 2014, p. 28). Returning to the example brought forth by Kaposy (2017), however, the point is that although we may embody an objectified "body two", it does not fully become who we are. Kaposy (2017) underscores the need to recognize the "anthropological constant" of bodily lived experience (body one) in the simulated clinical encounter. Although body one will never 'become' body two after long enough exposure, there is here a synthesis: "body one is situated within and permeated with body two, the cultural significations which we all experience." (Ihde, 2003, p. 13). Consequently, in VR, our "virtual selves" and virtual worlds - and how they are ontologically structureddo not become our new selves and our new worlds. They do, however, affect the way the "real world" and our "real selves" are constituted. Take for instance the study by Banakou et al. (2016), in which white participants were embodied in black avatars. The participants did not start to identify as black after the experiment and so radically change their sense of self. Yet, having experienced the world in which this was the structured ontology, their implicit racial bias, and so their subjectivity, was changed by means of the intervention. As Gualeni and Vella (2020) write: "in virtual worlds, human beings can reflect on their values and beliefs, take on new subjectivities, explore previously unexperienced ways of being, and take reflective stances toward their existence and their subjectivity in the actual world." (p. xix).

\section{The Relationship Between Environment and World}

In addition to considering the relationship between Human and User in the framework, it can be fruitful to clarify the relationship between the Environment and the World. In the phenomenological tradition, a given world is not understood as equivalent with reality. Rather, a world is understood as how reality is disclosed by human beings (Verbeek, 2005a). Worlds are-in their intentional relationship to human beingsintelligible, persistent, and "understood together" (Gualeni, 2015). The virtual environment with its "world characteristics" is seen as a part of the regular world in which it is accessible; however, engagement with it leaves the "real world" in the background in the alterity relation that is constituted. Ihde (2002) describes alterity as a "quasi-other or quasi-world with which the human actor relates" (p. 81). The virtual environment can be quite "other": it does not need to behave according to traditional ontologies and can instead, as we have seen, inverse them. In short, virtual environments are "fictive world[s] that [are] constructed, not copied" (Ihde, 2002, p. 81) and they come with their own "integrated ontology" (Metzinger, 2018, p. 4). The point is, however, that although the real and virtual worlds have distinct self-contained ontologies of their own, they are nevertheless highly interrelated. Again, we return to the concept of mediation. Postphenomenology stresses the role that technologies have in mediating humans' intentional relation toward their world, and in the case of Immersive VR, it is the experience of a virtually structured ontology that might reframe how humans disclose their worlds, and vice versa. Thus, postphenomenologically, we understand the ontologies of $\mathrm{VR}$ and RL as interrelated, so that experiencing a differently structured ontology in VR might affect the ontology of one's real world, or as Gualeni (2015) formulates it; “people's capability for structuring thought and rationalizing experience in relation to the actual world.” (p. 19). 


\section{The Scope of the Framework}

This paper has presented a theoretical framework for understanding the user-environment relations that Immersive VR gives rise to. One may ask, however, whether the framework extends to other VR technologies such as desktop VR (e.g., computer games), Mixed Reality (MR) technologies, and Augmented Reality (AR) technologies. The identified userenvironment relations we presented rests on the particular human-technology relation that VR constitutes: the possibility of embodying, as well as relating to as alterity, parts of the same technological mediator. Other immersive technologies, such as $\mathrm{AR}$ and $\mathrm{MR}$, do not constitute the same human-technology relation as VR. They are mainly distinguished in that they are not so immersive, and therefore engagement with the world persists actively instead of existing as a background relation. $\mathrm{MR}$, for instance, seem to constitute an immersion relation in the sense that the virtual is merged with the world, and so it is distinguished from Immersive VR in which there is not this "merging" of the physical and the virtual. AR technologies are also distinguished in the human-technology relation they constitute and are well described by Verbeek's augmentation relation. In the augmentation relation of a device such as the Google Glass, we embody the glasses, and we are in a hermeneutical relation to the technology, while our involvement with the world persists (Verbeek, 2015a).

The less immersive Desktop VR medium actually constitutes a similar relation to Immersive VR; the human interacts through an avatar toward the alterity of the virtual environment, where the world is in the background. Nevertheless, the experience is very different as Desktop VR is less immersive, and you can clearly see the bounds of the medium. The content is framed, and "[e]verything is in front of the participant" (Ihde, 2002 , p. 10). This framing restricts the medium's capability to encapsulate the user, and so the Desktop VR cannot achieve the same kind of mimesis with reality that Immersive VR can, where user interfaces can be natural and transparent, and the mediated information appears as if non-mediated. The degree of isomorphism between reality and virtuality that a simulating medium can achieve is important because it dictates how objects with their horizons and affordances are available to the user. For instance, Immersive VR can enable user interfaces to utilize natural bodily engagement with the virtual world (e.g., physically jumping vs. pressing space, or rotating head vs. moving mouse). This is not to say that desktop VR interfaces cannot also be embodied, or that all Immersive VR applications utilize natural interaction exclusively. In terms of general medium characteristics, however, desktop VR is not as inherently intuitive as immersive $\mathrm{VR}$ and may require more time to embody properly, just as we need to learn to drive a car before it truly becomes an extension of our bodies and we can pay attention to the road rather than how to maneuver the car.

To conclude, AR and MR constitute different humantechnology relations than Immersive VR, and so our framework of user-environment relations is not directly relevant for understanding user experience in environments using these technologies. The encapsulating capabilities of the Immersive
VR medium distinguishes it from other computer simulation technologies like Desktop VR, which do not leave the world in the background to the same extent as Immersive VR technologies. The capability of Immersive VR to provide realitybased interaction also contribute to the differences in how we experience worlds mediated through Immersive VR as opposed to Desktop VR.

\section{FUTURE WORK}

The perspective of postphenomenology sees the technological research product as a mediator that gives rise to a particular user-environment relation. It purports the view that the design of technological objects should also be understood as the design of human subjects. As Willis (2006) posits in her idea of ontological design, having this understanding-that what we design also designs us- "inevitably means undertaking any kind of designing activity with a very different kind of disposition." (p. 82). Developing systematic approaches of incorporating this understanding in evaluation and anticipation, however, is outside the scope of this paper. Future work addressing the applicability of post-phenomenological theory to concrete, practical cases would therefore complement our research. Here, we wish to highlight two avenues for research as particularly promising. Firstly, the development of systematic approaches to the empirical study of user experience in VR, and secondly, the development of guidelines for anticipating mediations as part of design processes and ethical assessment. For empirically studying user experience in VR, we see contextual inquiries where users are interviewed/queried in the virtual environment (Schwind et al., 2017, 2019; Alexandrovsky et al., 2021) as promising venture points for understanding userenvironment relations as such. In terms of anticipation, the post-phenomenological approach to "variational analysis" is highly relevant, which could be described as "brainstorming stabilities of a multi-stable technology" (ibid, p. 27). Rosenberger and Verbeek (2015) discuss how this approach is inspired by Husserl's eidetic reduction, but radically altered to find variations within particular contexts instead of the aim being to locate general "essences." Work looking into how postphenomenology's variational analyses can be performed more concretely for VR is here desirable.

Beyond the advancement of theoretical insights into methodology, however, what is most desired in future work is empirical insight into actual user-environment relations. In our analysis, we were not able to perform an analysis of the research participants' mediated experience, as in most of the cases, the participants' experiences were not outlined in-depth enough for it to be possible. Although assuming the participants' experienced the mediations as they were intended may be somewhat justified as the interventions were successful, we wish to stress that reaching experience through induction is not relating to actual, phenomenological accounts. In fact, the role of postphenomenology as we see it is precisely to move away from the researchers' assumptions of what experience is being mediated toward the actual mediated experience. 


\section{CONCLUSION}

Immersive VR is a remarkably flexible medium for interventions as it allows the construction of virtual worlds with ontologies radically different from the real world. Moving toward an understanding of the experiences underlying these effective interventions, we have proposed a theoretical framework that sees the user experience in Immersive VR as mediated in relations constituted between user and environment. The perspective that we advocate is distinguished from traditional approaches to understanding user experience in that it does not presuppose the human subject and the technology as poles between which interaction occurs. Rather, it sees the human subject and the experienced technology as a result of this interaction and the user experience as mediated in relations constituted between user and environment. We purport this perspective is a more relevant way of understanding the user experience underlying VR's capability to "change the self," as it specifically attends to how the human subject is mediated in the user-environment relation that is constituted. The applicability of the framework has been demonstrated through an analysis of a variety of VR interventions that constitute particular user-environment relations that vary greatly in terms of their ontological

\section{REFERENCES}

Achterhuis, H. (2001). American Philosophy of Technology: The Empirical Turn. Bloomington, IN: Indiana University Press.

Ahn, S. J., Le, A. M. T., and Bailenson, J. (2013). The effect of embodied experiences on self-other merging, attitude, and helping behavior. Media Psychol. 16, 7-38. doi: 10.1080/15213269.2012.755877

Ahn, S. J. G., Bostick, J., Ogle, E., Nowak, K. L., McGillicuddy, K. T., and Bailenson, J. N. (2016). Experiencing nature: embodying animals in immersive virtual environments increases inclusion of nature in self and involvement with nature. J. Comp. Mediat. Commun. 21, 399-419. doi: 10.1111/jcc4.12173

Alaraj, A., Lemole, M., Finkle, J., Yudkowsky, R., Wallace, A., Luciano, C., et al. (2011). Virtual reality training in neurosurgery: Review of current status and future applications. Surg. Neurol. Int. 2:52. doi: 10.4103/2152-7806.80117

Alexandrovsky, D., Putze, S., Schwind, V., Mekler, E. D. S., Smeddinck, J. D., Kahl, D., et al. (2021). "Evaluating user experiences in mixed reality," in CHI Conference on Human Factors in Computing Systems Extended Abstracts (New York, NY), 5.

Amores, J., Fuste, A., and Richer, R. (2019). "Deep reality: towards increasing relaxation in VR by subtly changing light, sound and movement based on HR, EDA, and EEG," in Extended Abstracts of the 2019 CHI Conference on Human Factors in Computing Systems (New York, NY), 1-2.

Ates, H. C., Fiannaca, A., and Folmer, E. (2015). "Immersive simulation of visual impairments using a wearable see-through display," in Proceedings of the Ninth International Conference on Tangible, Embedded, and Embodied Interaction (New York, NY), 225-228.

Aydin, C., González Woge, M., and Verbeek, P.-P. (2019). Technological environmentality: conceptualizing technology as a mediating milieu. Philos. Technol. 32, 321-338. doi: 10.1007/s13347-018-0309-3

Banakou, D., Beacco, A., Neyret, S., Blasco-Oliver, M., Seinfeld, S., and Slater, M. (2020). Virtual body ownership and its consequences for implicit racial bias are dependent on social context. R. Soc. Open Sci. 7:201848. doi: 10.1098/rsos.201848

Banakou, D., Hanumanthu, P. D., and Slater, M. (2016). Virtual embodiment of white people in a black virtual body leads to a sustained reduction in their implicit racial bias. Front. Hum. Neurosci. 10:601. doi: 10.3389/fnhum.2016.00601 structuring. Finally, we have discussed the interrelations of various aspects of our framework, addressed the framework's scope, and provided directions for future work in advancing the theoretical framework into the methodological.

\section{DATA AVAILABILITY STATEMENT}

The original contributions presented in the study are included in the article/supplementary material, further inquiries can be directed to the corresponding author/s.

\section{AUTHOR CONTRIBUTIONS}

The article was written by JV. The contributions by BW include supervision, comments, presentation, and discussion of conceptual ideas. All authors contributed to the article and approved the submitted version.

\section{FUNDING}

This research was funded by a Ph.D. stipend at the University of Bergen.

Banakou, D., Kishore, S., and Slater, M. (2018). Virtually being einstein results in an improvement in cognitive task performance and a decrease in age bias. Front. Psychol. 9:917. doi: 10.3389/fpsyg.2018.00917

Bourdin, P., Barberia, I., Oliva, R., and Slater, M. (2017). A virtual outof-body experience reduces fear of death. PLoS ONE 12:e0169343. doi: 10.1371/journal.pone.0169343

Çaliskan, O. (2011). Virtual field trips in education of earth and environmental sciences. Proc. Soc. Behav. Sci. 15, 3239-3243. doi: 10.1016/j.sbspro.2011.04.278

De Mul, J. (2010). Cyberspace Odyssey: Towards a Virtual Ontology and Anthropology. Newcastle upon Tyne: Cambridge Scholars Publishing.

Donohoe, J. (2017). "Introduction," in Place and Phenomenology, ed J. Donohoe (Washington, DC: RLI), 1-327.

Falconer, C. J., Slater, M., Rovira, A., King, J. A., Gilbert, P., Antley, A., et al. (2014). Embodying compassion: a virtual reality paradigm for overcoming excessive self-criticism. PLOS ONE 9:e111933. doi: 10.1371/journal.pone.0111933

Flobak, E., Wake, J. D., Vindenes, J., Kahlon, S., Nordgreen, T., and Guribye, F. (2019). "Participatory design of VR scenarios for exposure therapy," in Proceedings of the 2019 CHI Conference on Human Factors in Computing Systems (New York, NY), 1-12.

Fox, J., and Bailenson, J. N. (2009). Virtual self-modeling: the effects of vicarious reinforcement and identification on exercise behaviors. Media Psychol. 12, 1-25. doi: 10.1080/15213260802669474

Fox, J., Bailenson, J. N., and Tricase, L. (2013). The embodiment of sexualized virtual selves: the Proteus effect and experiences of self-objectification via avatars. Comput. Human Behav. 29, 930-938. doi: 10.1016/j.chb.2012. 12.027

Gonzalez-Franco, M., Slater, M., Birney, M. E., Swapp, D., Haslam, S. A., and Reicher, S. D. (2018). Participant concerns for the Learner in a Virtual Reality replication of the Milgram obedience study. PLoS ONE 13:e209704. doi: 10.1371/journal.pone.0209704

Groom, V., Bailenson, J. N., and Nass, C. (2009). The influence of racial embodiment on racial bias in immersive virtual environments. Social Influence 4, 231-248. doi: 10.1080/15534510802643750

Gualeni, S. (2015). Virtual Worlds as Philosophical Tools: How to Philosophize With a Digital Hammer. London: Palgrave Macmillan.

Gualeni, S., and Vella, D. (2020). Virtual Existentialism: Meaning and Subjectivity in Virtual Worlds. London: Springer Nature Switzerland, Palgrave Macmillan. 
Hamilton-Giachritsis, C., Banakou, D., Garcia Quiroga, M., Giachritsis, C., and Slater, M. (2018). Reducing risk and improving maternal perspective-taking and empathy using virtual embodiment. Sci. Rep. 8:2975. doi: 10.1038/s41598-018-21036-2

Hasler, B. S., Spanlang, B., and Slater, M. (2017). Virtual race transformation reverses racial in-group bias. PLOS ONE 12:e174965. doi: 10.1371 /journal.pone. 0174965

Hauser, S., Oogjes, D., Wakkary, R., and Verbeek, P.-P. (2018). "An annotated portfolio on doing postphenomenology through research products," in Proceedings of the 2018 Designing Interactive Systems Conference (New York, NY), 459-471.

Hershfield, H. E., Goldstein, D. G., Sharpe, W. F., Fox, J., Yeykelis, L., Carstensen, L. L., et al. (2011). Increasing saving behavior through age-progressed renderings of the future self. J. Mark. Res. 48, S23-S37. doi: 10.1509/jmkr.48.SPL.S23

Ihde, D. (1990). Technology and the Lifeworld: From Garden to Earth. Bloomington, IN: Indiana University Press.

Ihde, D. (2002). Bodies in Technology. Minneapolis, MN: University of Minnesota Press.

Ihde, D. (2003). Postphenomenology - Again? Available online at: https://sts.au. $\mathrm{dk} /$ fileadmin/sts/publications/working_papers/Ihde___Postphenomenology_ Again.pdf

Ihde, D. (2012). Experimental Phenomenology, 2nd Edn. Albany, NY: State University of New York Press.

Introna, L. (2017). "Phenomenological approaches to ethics and information technology," in Stanford Encyclopedia of Philosophy. Metaphysics Research Lab, Stanford University. Available online at: https://plato.stanford.edu/archives/ fall2017/entries/ethics-it-phenomenology

Jones, P. R., Somoskeöy, T., Chow-Wing-Bom, H., and Crabb, D. P. (2020). Seeing other perspectives: evaluating the use of virtual and augmented reality to simulate visual impairments (OpenVisSim). Npj Digital Med. 3, 1-9. doi: 10.1038/s41746-020-0242-6

Kaposy, C. (2017). "Postphenomenology of the robot medical student," in Postphenomenological Investigations: Essays on Human-Technology Relations, eds R. Rosenberger and P.-P. Verbeek (Lanham, MD: Lexington Books), 191-201.

Kay, A., and Goldberg, A. (1977). Personal dynamic media. Computer 10, 31-41. doi: 10.1109/C-M.1977.217672

Lanier, J. (2017). Dawn of the New Everything: Encounters With Reality and Virtual Reality, 1st Edn. New York, NY: Henry Holt and Co., Inc.

Leitner, J. B., Ayduk, O., Mendoza-Denton, R., Magerman, A., Amey, R., Kross, E., et al. (2017). Self-distancing improves interpersonal perceptions and behavior by decreasing medial prefrontal cortex activity during the provision of criticism. Soc. Cogn. Affect. Neurosci. 12, 534-543. doi: 10.1093/scan/nsw168

Lindner, P., Miloff, A., Fagernäs, S., Andersen, J., Sigeman, M., Andersson, G., et al. (2019). Therapist-led and self-led one-session virtual reality exposure therapy for public speaking anxiety with consumer hardware and software: A randomized controlled trial. J. Anxiety Disord. 61, 45-54. doi: $10.1016 /$ j.janxdis.2018.07.003

Loomis, J. M. (2016). Presence in virtual reality and everyday life: immersion within a world of representation. Presence Teleoperat. Virtual Environ. 25, 169-174. doi: 10.1162/PRES_a_00255

Madary, M., and Metzinger, T. K. (2016). Real virtuality: a code of ethical conduct. Recommendations for good scientific practice and the consumers of VR-technology. Front. Robot. AI 3, 1-23. doi: 10.3389/frobt.2016.00003

Maxhall, M., Backman, A., Holmlund, K., Hedman, L., Sondell, B., and Bucht, G. (2004). "Participants responses to a stroke training simulator," in 5th International Conference of Disability (Oxford), 225-229.

McCarthy, J., and Wright, P. (2004). Technology as Experience. Cambridge, MA: The MIT Press.

Merleau-Ponty, M. (2002). Phenomenology of Perception. London; New York, NY: Routledge.

Metzinger, T. K. (2018). Why is virtual reality interesting for philosophers? Front. Robot. AI 5, 1-19. doi: 10.3389/frobt.2018.00101

Milgram, S. (1964). Group pressure and action against a person. J. Abnorm. Soc. Psychol. 69, 137-143. doi: 10.1037/h0047759

Moran, S., Jäger, N., Schnädelbach, H., and Glover, K. (2016). ExoPranayama: a biofeedback-driven actuated environment for supporting yoga breathing practices. Pers. Ubiquitous Comp. 20, 261-275. doi: 10.1007/s00779-016-0910-3
Neyret, S., Navarro, X., Beacco, A., Oliva, R., Bourdin, P., Valenzuela, J., et al (2020). An embodied perspective as a victim of sexual harassment in virtual reality reduces action conformity in a later milgram obedience scenario. Sci. Rep. 10:6207. doi: 10.1038/s41598-020-62932-w

Nyre, L., and Vindenes, J. (2020). "Immersive journalism as witnessing," in Immersive Journalism as Storytelling: Ethics, Production, and Design, 1st ed, eds T. Uskali, A. Gynnild, S. Jones, and E. Sirkkunen (London: Routledge), 25-36.

Osimo, S. A., Pizarro, R., Spanlang, B., and Slater, M. (2015). Conversations between self and self as Sigmund Freud - a virtual body ownership paradigm for self counselling. Sci. Rep. 5:13899. doi: 10.1038/srep13899

Prpa, M., Cochrane, K., and Riecke, B. E. (2016). "Hacking alternatives in 21st century: designing a bio-responsive virtual environment for stress reduction," in Communications in Computer and Information Science, eds S. Serino, A. Matic, D. Giakoumis, G. Lopez, and P. Cipresso (Cham: Springer), 34-39.

Roo, J. S., Gervais, R., Frey, J., and Hachet, M. (2017). "Inner garden: connecting inner states to a mixed reality sandbox for mindfulness," in Proceedings of the 2017 CHI Conference on Human Factors in Computing Systems (New York, NY), 1459-1470.

Rosenberg, R. S., Baughman, S. L., and Bailenson, J. N. (2013). Virtual superheroes: using superpowers in virtual reality to encourage prosocial behavior. PLOS ONE 8:e55003. doi: 10.1371/journal.pone.0055003

Rosenberger, R., and Verbeek, P.-P. (2015). “A field guide to postphenomenology," in Postphenomenological Investigations: Essays on Human-Technology Relations, eds R. Rosenberger and P.-P. Verbeek (Lanham, MD: Lexington Books), 9-41.

Rua, H., and Alvito, P. (2011). Living the past: 3D models, virtual reality and game engines as tools for supporting archaeology and the reconstruction of cultural heritage - the case-study of the Roman villa of Casal de Freiria. J. Archaeol. Sci. 38, 3296-3308. doi: 10.1016/j.jas.2011.07.015

Satava, R. M. (1993). Virtual reality surgical simulator - the first steps. Surg. Endosc. 7, 203-205. doi: 10.1007/BF00594110

Schwind, V., Knierim, P., Haas, N., and Henze, N. (2019). "Using presence questionnaires in virtual reality," in Proceedings of the 2019 CHI Conference on Human Factors in Computing Systems (New York, NY), 1-12.

Schwind, V., Knierim, P., Tasci, C., Franczak, P., Haas, N., and Henze, N. (2017). "“'These are not my hands!": effect of gender on the perception of avatar hands in virtual reality," in Proceedings of the 2017 CHI Conference on Human Factors in Computing Systems (New York, NY), 1577-1582.

Seinfeld, S., Arroyo-Palacios, J., Iruretagoyena, G., Hortensius, R., Zapata, L. E., Borland, D., et al. (2018). Offenders become the victim in virtual reality: impact of changing perspective in domestic violence. Sci. Rep. 8:2692. doi: 10.1038/s41598-018-19987-7

Semertzidis, N. A., Sargeant, B., Dwyer, J., Mueller, F. F., and Zambetta, F. (2019). "Towards understanding the design of positive pre-sleep through a neurofeedback artistic experience," in Proceedings of the 2019 CHI Conference on Human Factors in Computing Systems (New York, NY), 1-14.

Slater, M. (2009). Place illusion and plausibility can lead to realistic behaviour in immersive virtual environments. Philos. Transac. R. Soc. B Biol. Sci. 364, 3549-3557. doi: 10.1098/rstb.2009.0138

Slater, M., Antley, A., Davison, A., Swapp, D., Guger, C., Barker, C., et al. (2006). A virtual reprise of the stanley milgram obedience experiments. PLoS ONE 1:e39. doi: 10.1371/journal.pone.0000039

Slater, M., Gonzalez-Liencres, C., Haggard, P., Vinkers, C., Gregory-Clarke, R., Jelley, S., et al. (2020). The ethics of realism in virtual and augmented reality. Front. Virtual Real. 1, 1-13. doi: 10.3389/frvir.2020.00001

Slater, M., and Sanchez-Vives, M. V. (2014). Transcending the self in immersive virtual reality. Computer 47, 24-30. doi: 10.1109/MC.2014.198

Slater, M., and Sanchez-Vives, M. V. (2016). enhancing our lives with immersive virtual reality. Front. Robot. AI. 3, 1-47. doi: 10.3389/frobt.2016. 00074

Sri Kalyanaraman, S., Penn, D. L., Ivory, J. D., and Judge, A. (2010). The virtual Doppelganger. J. Nerv. Mental Dis. 198, 437-443. doi: 10.1097/NMD.0b013e3181e07d66

Stepanova, E. R., Desnoyers-Stewart, J., Pasquier, P., and Riecke, B. E. (2020). "JeL: breathing together to connect with others and nature," in Proceedings of the 2020 ACM Designing Interactive Systems Conference (New York, NY), 641-654. 
Suzuki, K., Roseboom, W., Schwartzman, D. J., and Seth, A. K. (2017). A deepdream virtual reality platform for studying altered perceptual phenomenology. Sci. Rep. 7:15982. doi: 10.1038/s41598-017-16316-2

Tajadura-Jiménez, A., Banakou, D., Bianchi-Berthouze, N., and Slater, M. (2017). Embodiment in a child-like talking virtual body influences object size perception, self-identification, and subsequent real speaking. Sci. Rep. 7:9637. doi: 10.1038/s41598-017-09497-3

van Loon, A., Bailenson, J., Zaki, J., Bostick, J., and Willer, R. (2018). Virtual reality perspective-taking increases cognitive empathy for specific others. PLoS ONE 13:e0202442. doi: 10.1371/journal.pone.0202442

Verbeek, P.-P. (2005a). What Things Do (Philosophical Reflections on Technology, Agency, and Design), 1st Edn. University Park, MD: Penn State University Press.

Verbeek, P.-P. (2005b). "Beyond the human eye. Mediated vision and posthumanity," in Proceedings of AIAS Conference "Mediated Vision", eds P. J. H. Kockelkoren and P. Kockellkoren. (Veenman Publishers en ARTez Press), 1-7. Available online at: https://research.utwente.nl/en/publications/beyondthe-human-eye-mediated-vision-and-posthumanity

Verbeek, P.-P. (2011). Moralizing Technology: Understanding and designing the Morality of Things. Chicago, IL: University of Chicago Press.

Verbeek, P.-P. (2015a). "Designing the public sphere: information technologies and the politics of mediation," in The Onlife Manifesto, ed L. Floridi (Cham: Springer International Publishing), 217-227.
Verbeek, P.-P. (2015b). Cover story: beyond interaction: a short introduction to mediation theory. Interactions 22, 26-31. doi: 10.1145/27 51314

Verbeek, P. P. (2008). "Cyborg intentionality: Rethinking the phenomenology of human-technology relations," in Phenomenology and the Cognitive Sciences, ed S. Evan (Luxembourg: Springer Science \& Business Media B.V), 387-395.

Voordijk, H., and Vahdatikhaki, F. (2020). Virtual Reality learning environments and technological mediation in construction practice. Eur. J. Eng. Educ. 1-15. doi: 10.1080/03043797.2020.1795085

Willis, A.-M. (2006). Ontological designing. Design Philos. Pap. 4, 69-92. doi: $10.2752 / 144871306 X 13966268131514$

Conflict of Interest: The authors declare that the research was conducted in the absence of any commercial or financial relationships that could be construed as a potential conflict of interest.

Copyright $\odot 2021$ Vindenes and Wasson. This is an open-access article distributed under the terms of the Creative Commons Attribution License (CC BY). The use, distribution or reproduction in other forums is permitted, provided the original author(s) and the copyright owner(s) are credited and that the original publication in this journal is cited, in accordance with accepted academic practice. No use, distribution or reproduction is permitted which does not comply with these terms. 